\section{AB1403-HPR INVESTIGATION OF SPINE POSTURE, MOBILITY AND POSTURAL COMPETENCY IN LOW BACK PAIN PATIENTS WITH MODERATE TO SEVERE DISABILITY: A CASE-CONTROL STUDY}

Derya Ozer Kaya ${ }^{1}$, Seyda Toprak Celenay ${ }^{2} .{ }^{1} / z m i r$ Katip Celebi University, Department of Physiotherapy and Rehabilitation, Izmir, Turkey; ${ }^{2}$ Ankara Yildirim Beyazit University, Department of Physiotherapy and Rehabilitation, Ankara, Turkey

Background: Low back pain (LBP) is the most common musculoskeletal complaint especially in women that causing disability and growing as a global burden (1,2). Changes in spine structures and alignment have been important in the occurrence of LBP $(3,4)$.

Objectives: This study aimed to investigate spine posture, mobility and postural competency in women with and without LBP.

Methods: Thirty-four women with LBP having moderate to severe disability (age: 50.509 .26 years; body mass index (BMI): $34.765 .42 \mathrm{~kg} / \mathrm{m}^{2}$ ) and age-gender matched 37 asymptomatic healthy women (age: 48.948 .99 years; BMI: $31.976 .88 \mathrm{~kg} / \mathrm{m}^{2}$ ) were included. Oswetry Disability Index (ODI) was used for disability assessment (higher scores indicated more severe disability (0-50)). Overall spine posture, mobility, postural competency and spine check scores were evaluated using the Spinal Mouse (Idiag, Fehraltorf, Switzerland) device in standing position. The scores were evaluated between as poor "0" and "100" as perfect. T test was used for analysis.

Results: The mean score of ODI was 32.258.27 (moderate to severe disability). The scores of spine posture, mobility, postural competency and total spine check of women with LBP were found 24.7615.65; $25.1114 .60 ; 36.6421 .58 ; 30.2612 .06$, respectively; while, the scores of asymptomatic women were found 34.6716 .27 ; 34.4521.47; 38.2115.18; 38.7215.06, respectively. Lower posture $(p=0.011)$, mobility $(p=0.037)$ and total spine check scores $(p=0.011)$ were found in women with LBP compared to the controls; however, the postural competency was similar $(p=0.722)$.

Conclusion: Women with LBP had poor spine posture, mobility and total spine check scores, but similar postural competency in comparison to controls. In the clinics, considering these parameters might be important while planning the optimal treatment for LBP.

\section{REFERENCES}

[1] Hoy D, Bain C, Williams G, March L, Brooks P, Blyth F, Woolf A, Vos T, Buchbinder R. A systematic review of the global prevalence of low back pain. Arthritis Rheum 2012;64:2028-2037.

[2] Buchbinder R, van Tulder M, berg B, Costa LM, Woolf A, Schoene M, Croft P; Lancet Low Back Pain Series Working Group. Low back pain: a call for action. Lancet. 2018 9;391(10137):2384-2388.

[3] Alricsson M, Bjrklund G, Cronholm M, Olsson O, Viklund P, Svantesson U. Spinal alignment, mobility of the hip and thoracic spine and prevalence of low back pain in young elite cross-country skiers. J Exerc Rehabil. 2016;12(1):21-8.

[4] Misir A, Kizkapan TB, Tas SK, Yildiz KI, Ozcamdalli M, Yetis M. Lumbar spine posture and spinopelvic parameters change in various standing and sitting postures. Eur Spine J. 2018 Nov 29. doi: 10.1007/s00586-0185846-z.

Disclosure of Interests: None declared

DOI: 10.1136/annrheumdis-2019-eular.7324

\section{AB1404-HPR JIA TEAMWORK FOR IMPROVING PATIENTS' ACCEPTANCE OF INTRAARTICULAR GLUCOCORTICOID INJECTIONS IN LOCAL ANESTHESIA IN OUTPATIENT DEDICATED SERVICE}

Aurora Pucacco ${ }^{1}$, Hanan Jadoun ${ }^{1}$, Angela Aquilani ${ }^{1}$, Fabio Basta ${ }^{1}$, Andrea Uva ${ }^{2}$, Rebecca Nicolai ${ }^{1}$, Fabrizio De Benedetti ${ }^{1}$, Silvia Magni-Manzoni ${ }^{1} .{ }^{1}$ Ospedale pediatrico Bambino Ges, Roma, Italy, ${ }^{2}$ Sapienza University of Rome, Roma, Italy

Background: The discomfort and anxiety related to the medical care of patients with juvenile idiopathic arthritis (JIA), ampered by frequent and ongoing visits, diagnostic tests, medications and hospital stays, can negatively impact on the compliance during examinations and procedures, particularly intraarticular glucocorticoid injections (IAGI) in local anesthesia. A dedicated personnel for supporting patients and their caregivers may provide them relief, thus contributing also to a more serene environment for operators.

Objectives: To identify an appropriate approach for the relief of JIA patients most critical issues related to IAGI in local anesthesia. To provide preliminary validation of the approach identified.
Methods: In the first part of the study the nurse of the IAGI outpatient service at the study center had conversation with all consecutive JIA patients and caregivers seen at the service, while preparing them to IAG in local anesthesia and while on discharge. In agreement with the patient, the nurse synthetized in keywords and registered each patients most relevant feelings with regard to the IAGI procedure. Patients and caregivers were also asked to suggest feasible tools for a better acceptance of the procedure. The results were discussed within the JIA team, who selected in a questionnaire the most frequently reported keywords and identified the most feasible among the proposed supportive tools. Secondly, all consecutive JIA patients seen at the service were asked to complete the feeling-status questionnaire after the IAGI. As the tools were available, the questionnaire was completed after IAGI procedures tool-supported. Statistics included descriptive analysis and Students t-test for comparison between feelings rating by patients with and without supportive tools (significance: $p$-value $<0.05$ ).

Results: From the list of keywords obtained by the first 10 patients with the nurse, the most reported -stress, anxiety, fear, and anger- were included in a feeling questionnaire composed of a VAS 0-10 ( $0=$ none, $10=$ worst) for each keyword. Among the suggested supportive tools the JIA team identified as feasible: colored drawings/pictures in the procedure room and the availability of favourite songs/videoclips/cartoons on tablet just prior and during IAGI. Forthy-one patients completed the questionnaire in a mean time of 22 seconds. Among them, 24 (59\%) were supported by the selected tools, been meantime available, during IAGI procedure.

Abstract AB1404HPR Table 1. Mean of response to the feeling-questionnaire by JIA patients seen at the IAGI outpatient service without (A) and with (B) supportive tools

\begin{tabular}{|c|c|c|c|}
\hline & $\begin{array}{c}\text { Patients n.17 } \\
\text { (A) }\end{array}$ & $\begin{array}{c}\text { Patients } \\
\text { n.24 } \\
\text { (B) }\end{array}$ & P-value \\
\hline Female/Male & $16(94) / 1(6)$ & $\begin{array}{c}20(83) / \\
4(17)\end{array}$ & \\
\hline Age at IAGI, years, median (IQ range) & $\begin{array}{c}15,1(12,8- \\
20,5)\end{array}$ & $\begin{array}{l}13,5 \\
(9,7- \\
17,8)\end{array}$ & \\
\hline $\begin{array}{l}\text { Age at disease onset, years, median (IQ } \\
\text { range) }\end{array}$ & $4,9(1,7-11,2)$ & $\begin{array}{c}4,1(1,9 \\
-7,1)\end{array}$ & \\
\hline Stress $^{*}$, mean $(\mathrm{SD})$ & $9(1)$ & $4(2)$ & $<0.005$ \\
\hline Anxiety $^{*}$, mean (SD) & $9(1)$ & $4(2)$ & $<0.005$ \\
\hline Fear*, mean (SD) & $7(4)$ & $2(2)$ & $<0.005$ \\
\hline Anger*, mean (SD) & $5(3)$ & $1(2)$ & $<0.005$ \\
\hline
\end{tabular}

*VAS 0-10, 0=none, 10=worst

Conclusion: Our preliminary results highlight that a setting for IAGI more comfortable for patients provide an improvement in the JIA patients feelings rating undergoing injection procedures in local anesthesia. The nurses attitude to patients and the teamwork were fundamental in collecting the patients perspective and in adapting their suggestions to the IAGI outpatient service environment.

Acknowledgement: We acknowledge the Associazione Malattie Reumatiche Infantili (AMREI) Onlus for its support to the initiative

Disclosure of Interests: Aurora Pucacco: None declared, Hanan Jadoun: None declared, Angela Aquilani: None declared, Fabio Basta: None declared, Andrea Uva: None declared, Rebecca Nicolai: None declared, Fabrizio De Benedetti Grant/research support from: Abbvie, SOBI, Novimmune, Roche, Novartis, Sanofi, Pfizer, Silvia Magni-Manzoni Consultant for: Abbvie, Speakers bureau: Abbvie DOI: 10.1136/annrheumdis-2019-eular.3566

\section{AB1405-HPR REDUCED HAND FUNCTION AFFECTS ACTIVITY PERFORMANCE AND QUALITY OF LIFE IN PERSONS WITH SIBM}

Malin Regardt ${ }^{1,2}$. ${ }^{1}$ Karolinska Institute, LIME, Stockholm, Sweden; ${ }^{2}$ Karolinska University Hospital, Function area Occupational Therapy and Physiotherapy, Stockholm, Sweden

Background: Reduced hand function is common in persons with (IBM) (1). Information regarding how hand function deficits affect activities of daily living and quality of life is limited $(2,3)$. There is a need to improve treatment and increase knowledge on how to assess different aspects of sIBM 
Objectives: To investigate how hand function can influence activities of daily living and quality of life in persons with sIBM.

Methods: Data collection have been performed in both USA and in Sweden. In total 62 persons with IBM participated in this study (USA $n=36$, Sweden $n=26)$, median age were (IQR) $n=70$ (66-75) years with a median disease duration (IQR) 6 (2-8) year. The majority were men $\mathrm{n}=39(63 \%)$

Hand strength was measured with the Jamar dynamometer and dexterity by the Purdue Pegboard. Activity limitation measured by the Disability of the Arm, Shoulder and Hand (DASH) and Myositis Activities Profile (MAP). Quality of life by SF-36.

Results: Hand strength and dexterity in IBM was reduced in both women and men $(p<0.001)$. Percentage of reference values were (right hand/left hand) in Women $42 \% / 25 \%$, Men $30 \% / 27 \%$.

Persons with IBM had limitations in daily activities when compared to reference values $(p<0.001)$. The most limited activities were: Activities of moving around (extremely difficult), Recreation (Very difficult), Movement (moderately difficult) and Household activities (moderately difficult)

Persons with IBM had reduced quality of life in SF-36 domains; Physical Function, Role Physical, General Health, Vitality, Social Function and Mental Health.

Hand strength correlated moderately with activity limitation measures by DASH $(p<0.001)$ and MAP subscales; Movement, Activities of moving around, Personal hygiene, Housework, Social activities, Work/School and Recreation $(p<0.001)$ and moderately to SF-36-dimension Physical Function $(p<0.001)$.

Dexterity correlated moderately to DASH $(p<0.001)$ and all subscales of MAP $(p<0.001)$ and moderately to SF-36 dimensions Physical Function and Social Function $(\mathrm{p}<0.001)$.

Conclusion: Persons with IBM have reduced hand function and limitations in daily activities and quality of life. Suggesting the importance to include measures on both hand function, activity limitation and quality of life. The expertise on activity and occupational science of an occupational therapist may contribute to the assessment and treatment of persons with IBM.

\section{REFERENCES}

[1] Lindberg C, Oldfors A. Prognosis and prognostic factors in sporadic inclusion body myositis. Acta Neurol Scand. 2012;125(5):353-8.

[2] Eriksson M, Lindberg C. Hand function in 45 patients with sporadic inclusion body myositis. Occup Ther Int. 2012;19(2):108-16.

[3] Cox FM, Titulaer MJ, Sont JK, Wintzen AR, Verschuuren JJ, Badrising UA. A 12-year follow-up in sporadic inclusion body myositis: an end stage with major disabilities. Brain. 2011;134(Pt 11):3167-75.

Disclosure of Interests: None declared

DOI: 10.1136/annrheumdis-2019-eular.6854

\section{AB1406-HPR DIET AND LUPUS: WHAT DO THE PATIENTS THINK?}

George Robinson ${ }^{1,2}$, Thomas Mcdonnell ${ }^{2}$, Chris Wincup ${ }^{2}$, Lucia MartinGutierrez $^{1,2}$, James Wilton ${ }^{1}$, Anastasia Kalea ${ }^{3}$, Coziana Ciurtin ${ }^{1}$, Ines Pineda Torra ${ }^{4}$, Elizabeth Jury ${ }^{2}$, Jury Lab, Jury Lab. ${ }^{1}$ University College London, Centre for Adolescent Rheumatology, London, United Kingdom; ${ }^{2}$ University College London, Rheumatology, London, United Kingdom; ${ }^{3}$ University College London, Institute of Liver and Digestive Health, London, United Kingdom; ${ }^{4}$ University College London, Metabolism and Experimental Therapeutics, London, United Kingdom

Background: Cardiovascular disease (CVD) is the leading cause of mortality in patients with systemic lupus erythematosus (lupus). Therefore, using diet to control blood lipid levels and modify CVD risk could be a promising therapeutic strategy to control disease symptoms.

Objectives: The primary objective of this study was to learn about lupus patient experiences with diet including their opinion on considering diet as a therapeutic option. The secondary objective was to obtain this information in a cost and time effective manner.

Methods: A lay summary and a 15 question diet-based online survey was publicly available for 3 weeks. Social media was used to promote the survey through relevant charities, hospitals and research groups.

Results: 300 responses were received, 284 of whom had lupus. Patients reported that there was a lack of clinical counselling regarding diet with only $24 \%$ of patients stating that their doctor had spoken to them about diet. Despite this, $100 \%$ of patients stated that they would change their diet if they knew it would help their symptoms and $83 \%$ would take part in a future diet-based clinical trial. Text analysis of patient research suggestions identified a particular interest in using diet to treat fatigue and manage disease flares.

Conclusion: This project successfully gathered patient information regarding diet and lupus over a short timeframe using an anonymous social media platform. The survey provided evidence that patients support further research and potential diet intervention studies investigating the effect of diet on the symptoms of lupus.

\section{REFERENCES}

[1] Bernatsky S, Boivin JF, Joseph L, Manzi S, Ginzler E, Gladman DD, et al. Mortality in systemic lupus erythematosus. Arthritis \& Rheumatism. 2006:54(8):2550-7.

[2] McDonnell TCR, Wincup C, Rahman A, Giles I. Going viral in rheumatology: a rapid, cost-effective method of obtaining patient opinion about mechanistic research in SLE and APSA. Rheumatology. 2018;57:1.

Acknowledgement: Lupus UK, Rosetrees Trust, Versus Arthritis, Survey Monkey

Disclosure of Interests: None declared

DOI: 10.1136/annrheumdis-2019-eular.5669

\section{AB1407-HPR NEUROLOGICAL MANIFESTATIONS DURING THE BEHCET DISEASE}

Skouri Wafa ${ }^{1}$, sameh sayhi ${ }^{1}$, Najah Boussetta ${ }^{2}$, Rim Dhahri ${ }^{1}$, Faida Ajili ${ }^{3}$, Louzir Bassem ${ }^{4} .{ }^{1}$ military hospital, tunis, Tunisia; ${ }^{2}$ military hospital, tunis, Tunisia; ${ }^{3}$ autoimmune Disease Unit Research : UR17DN02, tunis, Tunisia; ${ }^{4}$ MILITARY HOSPITAL, tunis, Tunisia

Background: behcet disease $\mathrm{MB}$ is a systemic inflammatory disease whose common histopathological substratum is a vasculitis that can reach all vessels. Neurological impairment is one of the diagnostic criteria for MB. A serious condition affects the functional and vital prognosis.

Objectives: The aim of our work was to study the epidemiological, clinical, therapeutic and evolutionary characteristics of patients with BD with neurological involvement.

Methods: This was a retrospective, monocentric, descriptive study of NB from the adult population collected from a population of 150 patients monitored for MB. The study was conducted in the Neurology and Internal Medicine departments over a period of 19 years, from January 1997 to December 2015. Patients meeting the criteria of the international Study Group of the MB of 1990, those of the criteria of the International Criteria for Behcet Disease (ICBD) and the diagnostic criteria of Neuro-behcet defined proposed by the international consensus of experts of 2014 .

Results: We collect 35 patients. The gender ration was at 6 with 30 men for 5 women. The mean age of our population was 34+- 1.92 years. The neurological manifestations had inaugurated the MB in $55 \%$ of the cases. Ninety-four percent of patients had central nervous system involvement, while two patients had peripheral polyradiculoneuritis. Central involvement was parenchymal in $85 \%$ of cases, nonparenchymatous in $3 \%$ of cases and lixed in $6 \%$ of cases.

Cerebral imaging showed predominant demyelinating lesions in periventricular and brain stem in 15 patients, a peudo-tumor appearance in 3 patients, and vascular lesions with arterial aneurysm and cerebral thrombophlebitis in 2 patients. Therapeutically, all patients received corticosteroid therapy with immunosuppressive therapy for 15 patients. Three patients had received an immunomodulatory treatment of anti-TNF a Type. The evolution was by push in $60 \%$ of cases, primary progressive in $20 \%$ of cases and secondarily progressive in the rest of the cases. Three-quarters of patients with parenchymal brain injury had a favorable outcome. The Outcome was unfavorable in all patients with diffuse disease, brain stem damage, spinal cord injury, angio-behavior, polyradiculoneuropathy and mixed impairment. The change in visual impairment (NORB) was favorable in one case in two.

Conclusion: Neurological manifestations of MB are serious complications, and are typically poor prognosis both on a vital and functional level. Mortality remains high and neurological sequelae (motor and neuropsychological) are heavy. The prognosis in all the worse because the treatment is instituted late, hence the interest of an early and well conducted treatment

Disclosure of Interests: None declared

DOI: 10.1136/annrheumdis-2019-eular.8082 